\title{
Effect of Oral Administration of Weissella confusa on Fecal and Plasma Ethanol Concentrations, Lipids and Glucose Metabolism in Wistar Rats Fed High Fructose and Fat Diet
}

This article was published in the following Dove Press journal:

Hepatic Medicine: Evidence and Research

\author{
Fouad MF Elshaghabee' \\ Darab Ghadimi ${ }^{1}$ \\ Diana Habermann (D) \\ Michael de Vrese' \\ Wilhelm Bockelmann' \\ Hans-Jürgen Kaatsch (1D) ${ }^{2}$ \\ Knut J Heller $\mathbb{D}^{\prime}$ \\ Jürgen Schrezenmeir ${ }^{3,4}$
}

'Max Rubner-Institute, Federal Research Institute of Nutrition and Food,

Department of Microbiology and Biotechnology, Kiel, Germany; ${ }^{2}$ Institute of Legal Medicine, University Hospital of Schleswig-Holstein, Kiel, Germany; ${ }^{3}$ Max Rubner-Institute, Federal Research Institute of Nutrition and Food,

Department of Physiology and Biochemistry of Nutrition, Karlsruhe, Germany; ${ }^{4}$ Clinical Research Center, Kiel Innovation and Technology Center, Kiel, Germany
Correspondence: Darab Ghadimi

Tel +49 43160925I2

$\mathrm{Fax}+494316092472$

Email darab.ghadimi@mri.bund.de

\begin{abstract}
Background and Purpose: In previous investigations, Weissella confusa was shown to lack the metabolic pathway from fructose to mannitol and to produce ethanol when cultivated in the presence of fructose. Hence, we assessed the effect of oral administration of W. confusa (strain NRRL-B-14171) on blood and fecal ethanol concentrations, glucose and lipid metabolism and traits of the metabolic syndrome in Wistar rats $(n=27)$ fed diets with two different fat and fructose levels and with or without the addition of $W$. confusa during a total intervention time of 15 weeks (105 days).
\end{abstract}

Materials and Methods: From week 1 to 6, rats were given a medium fructose and fat (MFruMF) diet containing $28 \%$ fructose and $10 \%$ fat without the addition of $W$. confusa (control group, $\mathrm{n}=13)$ or mixed with $30 \mathrm{~g}$ per $\mathrm{kg}$ diet of lyophilized $W$. confusa $(10.56 \pm 0.20 \mathrm{log} \mathrm{CFU} / \mathrm{g}$; $W$. confusa group, $\mathrm{n}=14$ ). From week 7 to 15 , the percentage of dietary fructose and fat in the control and $W$. confusa group was increased to $56 \%$ and $16 \%$, respectively (high fructose-high fat (HFru-HF) diet).

Results: In HFru-HF-fed rats, $W$. confusa was detected in feces, regardless of whether $W$. confusa was added to the diet or not, but not in rats receiving the MFru-MF diet without added $W$. confusa or in an additional control group $(n=10)$ fed standard rat food without fructose, increased fat content and $W$. confusa. This indicates that fecal $W$. confusa may be derived from orally administered $W$. confusa as well as - in the case of high fructose and fat intake and obesity of rats - from the intestinal microbiota. As shown by multifactorial ANOVA, blood ethanol, the relative liver weight, serum triglycerides, and serum cholesterol as well as fecal ethanol, $\mathrm{ADH}$, acetate, propionate and butyrate, but not lactate, were significantly higher in the $W$. confusa - compared to the control group.

Discussion: This is the first in vivo trial demonstrating that heterofermentative lactic acid bacteria lacking the mannitol pathway (like $W$. confusa) can increase fecal and blood ethanol concentrations in mammals on a high fructose-high fat diet. This may explain why $W$. confusa resulted in hyperlipidemia and may promote development of NAFLD in the host. Keywords: high fructose-high fat diet, fecal ethanol, fecal short-chain fatty acids, lipidemia, glycemia, NAFLD

\section{Introduction}

Prevalence of obesity and related risks of metabolic disorders such as fatty liver, dyslipidaemia, type 2 diabetes and cardiovascular disease are increasing in industrial and developing countries representing a global metabolic health burden. ${ }^{56}$ 
Non-alcoholic fatty liver diseases (NAFLD) are defined as fat accumulation in the liver and a liver weight exceeding $5 \%$ to $10 \%$ of body weight. Lipid storage in hepatocytes (hepatic steatosis) and inflammation are associated with nonalcoholic steatohepatitis (NASH) which is characterised by macrovesicular steatosis, lobular inflammation and hepatocellular ballooning in addition to steatosis. ${ }^{51}$

Gut microbiota may be involved in different mechanisms of pathogenesis of NAFLD and further traits of the metabolic syndrome by increasing production of endogenous ethanol from fructose, by activation of inflammatory mechanisms via translocation of various bacterial components, mainly lipopolysaccharide (LPS), via a reduced intestinal barrier and increased energy metabolism. ${ }^{57,65}$ In rats, counts of $E$. coli were increased in the small intestinal microbiota of a non-alcoholic steatohepatitis (NASH) group on a high-fat diet in comparison with a control group on normal chow. ${ }^{69}$

Studies of the meta-proteome and metabolome of mice fed a high fat (HF) diet demonstrated that this diet markedly affects the gut bacterial ecosystem at the functional and physiological levels. Hormonal and microbial networks, bile acid and bilirubin metabolism, and amino acid and simple sugars metabolism were altered when mice were fed HF diets. ${ }^{22}$

Beside HF diet, high-fructose diet is associated with obesity, insulin resistance and other traits of the metabolic syndrome. ${ }^{13,26,42,43}$ Feeding mice C57BL/6 with a highfructose diet caused inflammation of liver tissue by increasing intestinal translocation of endotoxin. ${ }^{8}$ Fructose represents a key player in the development of NAFLD. In Wistar rats, plasma alanine aminotransferase and triglycerides did not differ between rats fed for 5 weeks a diet containing either high concentrations of fat $(15 \%)$ and high fructose $(50 \%)$ or corn starch. Histopathological examination, however, showed macrovesicular steatosis in rats fed HF-HFru diet in comparison with the corn starch control group. ${ }^{42}$

Weissella confusa is a Leuconostoc-like, Gram-positive, catalase-negative, non-motile, short rod-shaped microorganism belonging to firmicutes, ie lactic acid-producing bacteria. It has been isolated from clinical and human fecal samples, dairy and meat products and can be used as adjunct starter culture. ${ }^{2,4,9,34,67}$ Bacteria of the genus Weissella do not possess cytochromes and ferment glucose heterofermentatively via the hexose-monophosphate and phosphoketolase pathways. End products of glucose heterofermentation include lactic acid (with some species producing only
D (-) and others both $\mathrm{D}(-)$ and $\mathrm{L}(+)$ lactic acid enantiomers), gas $\left(\mathrm{CO}_{2}\right)$ and ethanol and/or acetate. ${ }^{10,19,34}$ Within the genus, Weissella species were described, which produce mannitol from fructose, as well as species which were not able to produce mannitol from fructose. The species W. confusa did not produce mannitol from fructose in all studies published so far. ${ }^{27,35,42}$

In previous work, $W$. confusa strain NRRL-B-14171, which is not able to metabolize fructose via the mannitol pathway, was found to produce ethanol and lactate in about equimolar amounts from the fermentation of fructose in single culture. ${ }^{27}$ The present study aims at clarifying whether orally administrated $W$. confusa can modulate fecal microbial metabolites (particularly ethanol), can increase ethanol blood levels and can affect glucose and lipid metabolism in rats fed a high-fructose diet.

\section{Materials and Methods Preparation of Lyophilized W. confusa}

$W$. confusa was obtained from Northern Regional Research Laboratory (NRRL), Peoria, USA as L. reuteri NRRLB-14171; however, 16S rDNA sequencing clearly identified it as $W$. confusa. $W$. confusa was propagated in the medium for colonic bacteria supplemented with $1.5 \%$ sodium citrate $(\mathrm{MCB}-\mathrm{C})^{63}$ and incubated anaerobically in an anaerobic chamber (MAKS MG Co., Don Whitley Scientific Limited, West Yorkshire, England) at $37^{\circ} \mathrm{C}$ for $18 \mathrm{~h}$. Cells from MCB-C cultures were harvested by centrifugation $\left(6371 \mathrm{x} \mathrm{g}, 4^{\circ} \mathrm{C}, 10\right.$ min), washed once with phosphate-buffered saline (PBS), concentrated 10 times in sterilized skimmed milk and kept at $-65^{\circ} \mathrm{C}$ in glass bottles for $18 \mathrm{~h}$. Lyophilization of concentrated cells was performed in Lyovac ${ }^{\mathrm{TM}}$ GT2 equipment (Amsco/ Finn-Aqua, Wommelgem, Belgium) for $72 \mathrm{~h}$. The number of viable cells before and after lyophilization was determined by counting on MRS agar (Merck Co., Darmstadt, Germany). The viable count of lyophilized $W$. confusa was $10.56 \pm 0.20 \mathrm{log}$ $\mathrm{CFU} / \mathrm{g}$. The range of water of activity $\left(\mathrm{A}_{\mathrm{w}}\right)$ value was 0.013 to 0.021 (HygroLab, Rotronic Instrument Corp., Hauppauge NY, USA) in lyophilized samples.

\section{Animals and Feeding Protocol}

Male Wistar rats (Wistar Han IGS, Strain code: 273 Charles Rivers, Sulzfeld, Germany), 3-weeks old (50 g), were housed in microisolator plastic cages individually $\left(\mathrm{n}=1\right.$ per cage) and maintained on Ssniff ${ }^{\circledR}$ NR pellets $\left(\right.$ Ssniff $^{\circledR}$, Soest, Germany; Table 1) and water ad libitum 
Table I Composition of the Diets Used

\begin{tabular}{|c|c|c|c|c|}
\hline Contents Diet & I. (Ssniff NR) & II. LFru-LF & III. MFru-MF & IV. HFru-HF \\
\hline Product code* & VI444-0 & S83I8-E00I & Mix of II+IV & S83I8-E003 \\
\hline \multicolumn{5}{|l|}{ Crude nutrients (g nutrient/ $100 \mathrm{~g}$ diet) } \\
\hline Protein (casein; $\mathrm{N} \times 6.25$ ) [\%] & 26.1 & 17.6 & 17.6 & 17.6 \\
\hline Starch [\%] & 26.9 & 53.8 & 26.9 & - \\
\hline Fructose [\%] & - & - & 28.0 & 56.0 \\
\hline Cellulose powder [\%] & 3.7 & 15.0 & 9.0 & 3.0 \\
\hline Porc lard [\%] & - & - & 6.0 & 12.0 \\
\hline Other fat [\%] & 5.8 & & 4.0 & 4.0 \\
\hline Vitamin + mineral mix [\%] & 5.0 & 5.0 & 5.0 & 5.0 \\
\hline \multicolumn{5}{|l|}{ Metabolizable energy [MJ ME/kg diet] } \\
\hline Total ME $[\mathrm{MJ} / \mathrm{kg}]$ & 14.3 & 13.8 & 16.2 & 18.7 \\
\hline Thereof from carbohydrates [\%] & 45 & 67.4 & 59.9 & 51.7 \\
\hline Lipids [\%] & 14 & 11.3 & 21.9 & 32.5 \\
\hline Protein [\%] & 41 & 21.3 & 18.6 & 15.8 \\
\hline
\end{tabular}

Note: *(Product code) Given by the diet provider (Ssniff, Soest, Germany).

Abbreviations: Ssniff NR, maintenance diet for nude rats; LFru-LF, low fructose-low fat diet; MFru-MF, medium fructose-medium fat diet; HFru-HF, high fructose-high fat diet; $\mathrm{N}$, measured nitrogen content of the diet; MJ, megajoule; ME, metabolizable energy.

for 8 weeks, until they had reached 290-355 g, in ambient temperature and humidity with a $12 \mathrm{~h}$ light-dark cycle. Thereafter, rats were randomly allocated to two groups. In the $W$. confusa group, the diet was mixed with lyophilized W. confusa (0.32 log CFU/kg diet according to Fukushima and Nakano, 1996), ${ }^{32}$ in the control group $(\mathrm{n}=13)$, the diet was given without $W$. confusa. In the first 6 weeks of dietary intervention, rats were fed medium fructosemedium fat (MFru-MF) diet (28\% fructose, $10 \%$ fat; Table 1).

After 6 weeks, ie from week 7 to 15, control and $W$. confusa rats were fed a high fructose and fat (HFruHF) diet containing 56\% fructose and $16 \%$ fat (Table 1) without or with $0.32 \log \mathrm{CFU} / \mathrm{kg} W$. confusa.

In parallel, further 10 rats received a standard diet for (nude) rats (Ssniff $^{\circledR}$ NR V1444-0; Table 1) for 15 weeks, in order to investigate whether fructose and/or increased fat content in the diet induce a shift in gut microbiota. Figure 1 shows the feeding scheme.

All diets (Table 1) were purchased from Ssniff ${ }^{\circledR}$, Soest, Germany. The MFru-MF diet was produced by mixing the HFru-HF diet (Ssniff ${ }^{\circledR}$ S8318-E003) with a low fat and fructose diet (LFru-LF; Ssniff ${ }^{\circledR}$ S8318-E001).

Animal care and experimentation were performed in agreement with the Guide for Care and Use of Laboratory Animals of the National Institutes of Health $(\mathrm{NIH})$ and in accordance with the EEC directive of $1986(86 / 609 / \mathrm{EEC})$ and were approved by the ethical committee of the Ministry for Agriculture, the Environment and Rural Areas of Schleswig-Holstein, Germany.

\section{Blood Collection}

For control, whole blood samples were taken from the retinal vein of the rats after 2 and 6 weeks on MFru-MF diet. For this, animals were anaesthetized by intraperitoneal injection of ketamine and xylazine $(0.25 \mathrm{~mL} / 100 \mathrm{~g}$ body weight; mixed in the ratio $4: 1$ ).

For animal welfare reasons, to minimize the distress imposed on the rats (pain, suffering), control blood samples were taken only from some randomly selected animals each: after 2 weeks of intervention from seven rats of the control group and five rats of the $W$. confusa group and after 6 weeks from five rats of each group (Table 2). If retrobulbar blood sampling had not yielded enough blood in an animal, blood collection was not repeated. This contributed to an unbalanced study design, that means, in the statistical data analysis by multifactor ANOVA, unequal numbers of observations at the various factor levels had to be considered.

At the end of the experiment (day 106), rats were anaesthetized as described above and killed by bleeding. Blood was collected and plasma was immediately separated by centrifugation ( $4000 \mathrm{x} \mathrm{g}$ for $10 \mathrm{~min}$ ) and stored 


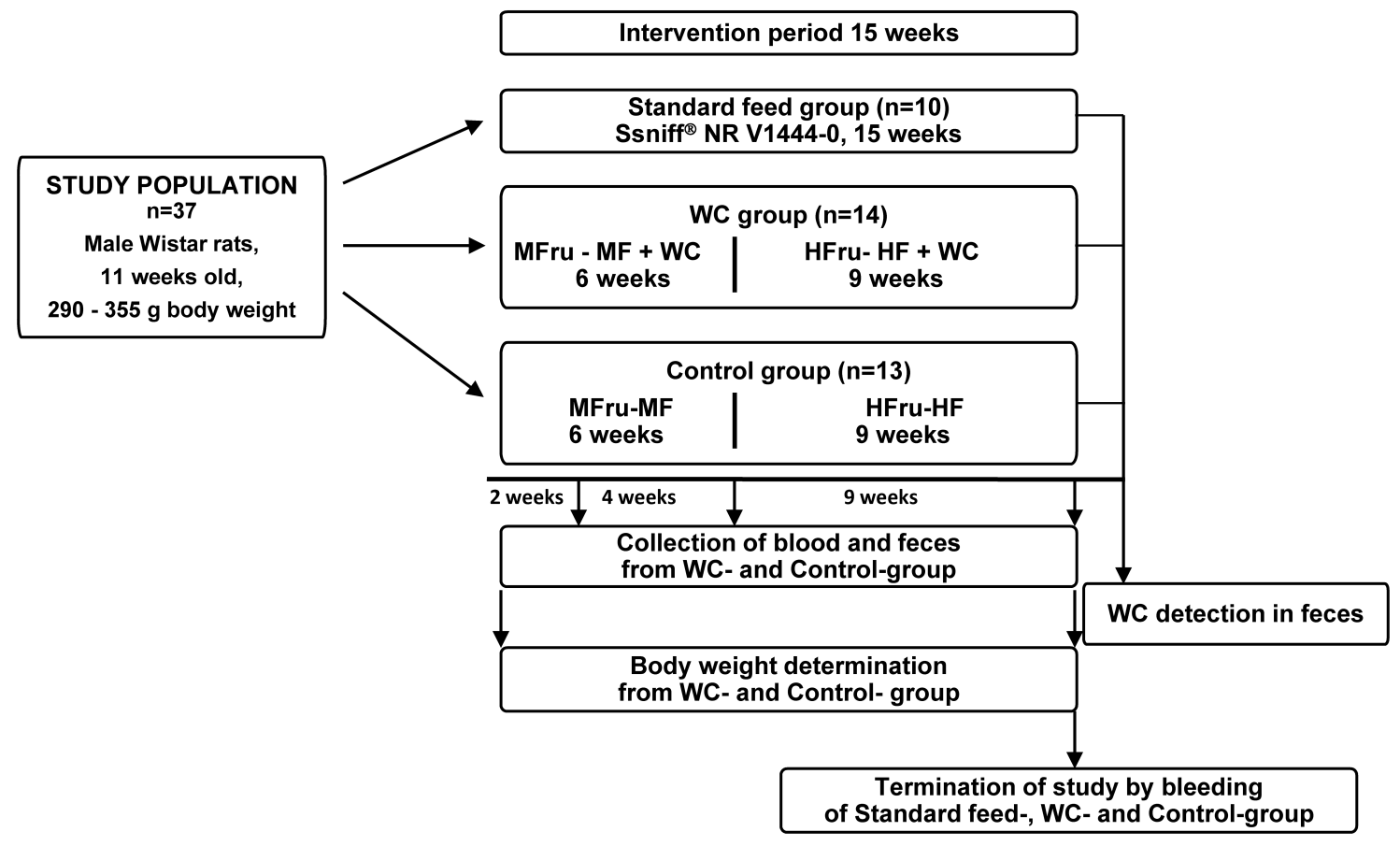

Figure I Feeding scheme.

at $-20^{\circ} \mathrm{C}$ until analysis. For assessing liver parameters, using sterile scissors and tweezers, the abdomen was opened and the entire liver was dissected under sterile conditions. For fecal microbial analysis, the whole colon of the rats was removed under sterile conditions, transferred immediately to sterile $50 \mathrm{~mL}$ tubes, placed in an anaerobic jar containing Oxoid ${ }^{\mathrm{TM}}$ anaerobic gas generator, and immediately transported to the microbiology laboratory.

\section{Determination of Biochemical Parameters in Blood Samples}

Serum alanine transaminase (ALT), aspartate aminotransferase (AST), triglycerides (TG), cholesterol (Thermo Fisher Scientific, Passau, Germany) and plasma glucose (New Blood Sugar Test, Boehringer Mannheim, Germany) were determined on a Konelab 20i clinical chemistry analyser (Kone, Helsinki, Finland) as described by the manufacturers. Ethanol in blood was measured by gas chromatography (GC). Briefly, $100 \mu \mathrm{L}$ of blood samples was mixed with $3 \mathrm{~g}$ sodium sulfate and $400 \mu \mathrm{L}$ t-butanol in GC-vials; D $=20 \mathrm{~mm}$ (Uwe Neuber analytic supply and service, Stelle, Germany). GC system consisted of gas chromatograph Clarus 580, Turbomatrix 110 headspacesampler and Elite-wax column, $30 \mathrm{~m} / 0.32 \mathrm{~mm} / 0.5 \mu \mathrm{m}$ (Perkin-Elmer Co., Massachusetts, USA).

\section{Determination of Hepatic Index}

The hepatic index (HI\%) at the end of the intervention period was calculated according to the equation of Yang (2001) as $\mathrm{HI} \%$ = weight of liver/weight of body $\times 100 .^{72}$

\section{Determination of Fecal Short-Chain Fatty Acids (SCFAs) and Ethanol}

A specimen of $0.1 \mathrm{~g}$ fresh feces from each rat was suspended in $1 \mathrm{~mL}$ phosphate buffer saline ( $\mathrm{pH} 7)$, containing $0.05 \%$ cysteine-HCL and $0.2 \%$ Tween 80 as emulsifier to obtain homogenized fecal slurries. Samples were homogenized and centrifuged at $14,000 \mathrm{Xg}$ and $4^{\circ} \mathrm{C}$ for $15 \mathrm{~min}$, the clear supernatant was separated and filtered by a membrane filter with $0.4 \mu \mathrm{m}$ pores. All supernatant samples were frozen at $-20^{\circ} \mathrm{C}$ until analysis. The analysis of ethanol and short-chain fatty acids (acetate, butyrate and propionate) was determined by HPLC as described previously. $^{27}$

\section{Extraction of DNA Using QIAamp DNA Stool Kit}

A specimen of feces from each rat was weighted and homogenized in 7-10 volumes of stool lysis buffer (Buffer ASL). $1.5 \mathrm{~mL}$ of the slurry was incubated at $95^{\circ} \mathrm{C}$ for $5 \mathrm{~min}$. After 
Table 2 Effects of WC NRRL-B- $|4| 7 I^{\dagger}$ and of a Medium or High Fat and Fructose Content in the Diet on Body Weight, Parameters of Host Metabolism and Microbial Metabolites in Wistar Rats

\begin{tabular}{|c|c|c|c|c|c|c|c|c|}
\hline \multirow[t]{2}{*}{ Parameter } & \multicolumn{3}{|c|}{ Control Group (Without WC) } & \multicolumn{3}{|c|}{ WC Group (+ WC) } & \multirow{2}{*}{$\begin{array}{l}\text { WC vs Control } \\
\mathrm{P}_{\mathrm{vc}}^{\#}\end{array}$} & \multirow{2}{*}{$\begin{array}{l}\text { Impact of Fruct }+ \text { Fat } \\
P_{d}\end{array}$} \\
\hline & $\mathbf{n}_{\mathbf{c}}$ & LSm & SE & $\mathbf{n}_{\mathbf{c}}$ & LSm & SE & & \\
\hline \multicolumn{9}{|l|}{ A) Body parameters } \\
\hline $\mathrm{BW}[g]$ & 43 & 390.6 & 5.1 & 45 & 388.9 & 4.9 & 0.811 & $0.000 * *$ \\
\hline BW (week I5) ${ }^{\dagger \dagger}[g]$ & 13 & 462.4 & 11.3 & 14 & 450.0 & 6.9 & 0.277 & n.m. ${ }^{\ddagger \ddagger}$ \\
\hline \multicolumn{9}{|l|}{ B) Liver parameter } \\
\hline Hepatic index [\%] & 13 & 3.008 & 0.092 & 14 & 3.293 & 0.089 & $0.036^{*}$ & n.m. \\
\hline AST [U/L] & 26 & 95.02 & 11.87 & 25 & 83.15 & 12.10 & 0.487 & 0.629 \\
\hline ALT [U/L] & 26 & 40.58 & 2.968 & 25 & 41.10 & 3.027 & 0.903 & 0.205 \\
\hline \multicolumn{9}{|c|}{ C) Blood parameters ( $\mathrm{P}=$ sodium fluoride plasma; $\mathrm{s}=$ serum; $\mathrm{b}=$ blood) } \\
\hline pGlucose $[\mathrm{mg} / \mathrm{dL}]$ & 14 & 358.9 & 17.67 & 15 & 358.2 & 17.07 & 0.980 & n.m. \\
\hline sTG [mg/dL] & 27 & 263.9 & 29.25 & 25 & 335.5 & 30.40 & $0.097^{\S}$ & $0.002 * *$ \\
\hline sCholesterol $[\mathrm{mg} / \mathrm{dL}]$ & 13 & 83.95 & 3.414 & 14 & 99.39 & 3.290 & $0.003^{* *}$ & n.m. \\
\hline bEthanol $[\mu \mathrm{g} / \mathrm{mL}]$ & 13 & 0.115 & 16.28 & 14 & 75.07 & 15.69 & $0.003^{* *}$ & n.m. \\
\hline \multicolumn{9}{|c|}{ D) Fecal (F) parameters ( $\mathrm{I} \mathrm{mL}$ in the concentration units correspondents to $0.1 \mathrm{~g}$ fresh feces) } \\
\hline fEthanol $[\mu \mathrm{g} / \mathrm{mL}]$ & 43 & 53.19 & 20.62 & 43 & 155.2 & 20.62 & $0.001 * *$ & $0.023^{*}$ \\
\hline fADH [U/L] & 45 & 3.233 & 0.056 & 45 & 3.994 & 0.056 & $0.000 * *$ & $0.000 * *$ \\
\hline fAcetate $[\mu \mathrm{g} / \mathrm{mL}]$ & 43 & 668.7 & 83.82 & 44 & 1268.7 & 82.87 & $0.000 * *$ & $0.000 * *$ \\
\hline fPropionate $[\mu \mathrm{g} / \mathrm{mL}]$ & 43 & 292.1 & 42.26 & 44 & 539.6 & 41.78 & $0.000 * *$ & $0.025^{*}$ \\
\hline fButyrate $[\mu \mathrm{g} / \mathrm{mL}]$ & 43 & 192.3 & 43.20 & 44 & 400.8 & 42.71 & $0.001 * *$ & $0.000 * *$ \\
\hline flactate $[\mu g / m L]$ & 43 & 18.43 & 10.38 & 44 & 39.62 & 10.26 & 0.151 & 0.144 \\
\hline \multicolumn{9}{|c|}{ E) Detection of Weissella in rat feces } \\
\hline Week 15 (Ssniff NR) & - & & & & & & & \\
\hline Week 6 (MFru-MF) & - & & & + & & & & \\
\hline Week I5 (HFru-HF) & + & & & + & & & & \\
\hline
\end{tabular}

Notes: ${ }^{\dagger}$ Addition of $10.56 \pm 0.20 \log$ CFU per g diet of lyophilized WC. ${ }^{\dagger}$ Weight (week 15) is the (arithmetic) mean weight at the end of the trial. *Significant effect; **Highly significant effect; ${ }^{\S}$ Significance on one-sided statistical testing. ${ }^{*} \mathrm{P}$-value of the comparison of the WC vs control group, p-values below 0.05 are considered significant. ${ }^{\# \#} \mathrm{P}$-value of the dose effect of fructose and fat, ie medium versus high fructose and fat diet, $\mathrm{p}$-values below 0.05 are considered significant. Ssniff NR (Product name) given by the provider (Ssniff, Soest, Germany).

Abbreviations: WC, Weissella confusa NRRL-B-1417I; fruct, fructose; $n_{c}$, number of measured cases; LSm, least-squares means; SE, standard error; BW, body weight; n.m., not measured/measurable; AST, aspartate aminotransferase; ALT, alanine aminotransferase; TG, triglycerides; U/L, units per liter; ADH, alcohol dehydrogenase; Ssniff NR, maintenance diet for nude rats; MFru-MF, medium fructose-medium fat diet; HFru-HF, high fructose-high fat diet; vs, versus.

$15 \mathrm{~s}$ of vortexing, the sample was centrifuged for $1 \mathrm{~min}$ at $20,000 \times \mathrm{g}$, room temperature (RT) to pellet stool particles. Up to $1.2 \mathrm{~mL}$ of supernatant was transferred into a new centrifuge tube. Half an InhibitEX ${ }^{\mathrm{TM}}$ tablet was added, followed by vortexing for $1 \mathrm{~min}$ until the tablet was completely suspended. Extraction of DNA from stool samples was performed according to the manual of QIAGEN GmbH (Hilden, NRW, Germany). For elution, the QIAamp spin column was transferred into a $1.5 \mathrm{~mL}$ centrifugation tube and $200 \mu \mathrm{L}$ Buffer $\mathrm{AE}$ (equilibrated at $70^{\circ} \mathrm{C}$ ) were added. After 1 min incubation at RT, DNA was eluted by centrifugation at $20,000 \mathrm{x}$ g for $1 \mathrm{~min}$. The samples were stored in aliquots of $50 \mu \mathrm{L}$ at $-20^{\circ} \mathrm{C}$.

\section{Detection of W. confusa NRRL-B-|4|7| in Rat Feces}

Amplification of $W$. confusa specific gene in rat feces was carried out using IQ5 Thermocycler (BioRad Co., California, USA) and the specific primers BP40F (5'-GGCGGATT GGTCTCTTTTTG-3') and BP264R (5'-CACGCTCAGTA ACCGTGTGC-3' $)^{33}$ resulting in amplification of a $225 \mathrm{bp}$ fragment. The reaction mixture $(25 \mu \mathrm{L})$ contained $10 \mu \mathrm{L}$ 5Prime Mastermix (5Prime), $10 \mathrm{pmol} / \mu \mathrm{L}$ of each primer, $0.025 \mu \mathrm{g}$ of bovine serum albumin and $5 \mu \mathrm{L}$ of rat feces DNA solution. In a control reaction, $2.3 \mathrm{ng}$ genomic DNA of W. confusa NRRL-B-14171 was used. The amplification 
program was $95^{\circ} \mathrm{C}$ for $5 \mathrm{~min} ; 35$ cycles of $95^{\circ} \mathrm{C}$ for $30 \mathrm{~s}$, $60^{\circ} \mathrm{C}$ for $30 \mathrm{~s}$, and $72^{\circ} \mathrm{C}$ for $30 \mathrm{~s}$; and $72^{\circ} \mathrm{C}$ for $30 \mathrm{~s}$; and, finally $72^{\circ} \mathrm{C}$ for $10 \mathrm{~min}$. Polymerase chain reaction (PCR)fragments were analyzed by gel electrophoresis $(2 \% \mathrm{w} / \mathrm{V}$ Agarose).

\section{Sample Size Determination and Randomization}

Since no statistically useable quantitative data were available for the effect of oral administration of $W$. confusa on ethanol synthesis from fructose and its effects on fecal and blood ethanol concentrations and parameters of the metabolic syndrome in rats, no statistically reliable sample size calculation could be performed. Instead, the number of animals required was estimated on the basis of earlier trials with high fructose and fat-fed rats.

Based on this, we assumed a required sample size of 14 rats per group (plus $2 \times 3$ animals to compensate for possible rat losses during the trial), so that a total of 34 six-week-old rats were randomly distributed to the control and experimental groups with computer-generated random numbers.

\section{Statistical Analysis}

For statistical analysis of (i) the effect of $W$. confusa on hepatic markers and selected parameters of the metabolic syndrome in HFru-HF diet-fed rats and (ii) a dose effect of the fat and fructose allowance, a two-way repeatedmeasures ANOVA as a special case of the multifactor ANOVA procedure of the software package "Statgraphics Plus for Windows" (version 4.5, Manugistics, Rockville, MD, USA) was applied.

"Group" (with the two-factor levels "addition or no addition of $W$. confusa to the diets") was used as the categorical variable and "supply" (with the levels "medium" $=28 \%$ fructose and $10 \%$ fat versus "high" $=56 \%$ fructose plus $16 \%$ fat) were used as a covariate. The levels of the categorical variable were measured in different rat groups, while in the covariate, all levels were measured in the same rat. Because of this design, in Table 2 in the $W$. confusa and control group, the Least Square (LS) means are used instead of arithmetic means; both differ somewhat from each other only in the case of unequal numbers of observations at the various factor levels. For parameters for which a sufficient number of samples were not available and/or which were determined only at the end of the experiment, because sampling required killing of the animals, the dose effect could not be measured.

Besides LS means $\pm \mathrm{SD}$ the procedure provides the $\mathrm{p}$-values of the effects of $W$. confusa $\left(\mathrm{p}_{\mathrm{vc}}\right)$ and of the fat and fructose supply $\left(\mathrm{p}_{\mathrm{d}}\right)$ on the parameters. Moreover, $\mathrm{n}_{\mathrm{c}}$ in Table 2 represents the number of measured cases. Since the two-way repeated-measures ANOVA also includes in statistical evaluation the data from samples collected before the end of the study, $\mathrm{n}_{\mathrm{c}}$ is only identical with the number of evaluated animals per group if samples were taken only at the end of the experiment.

\section{Results}

The effects of the administration of $W$. confusa and a medium or high fat plus fructose allowance on weight development, hepatic markers and a number of other parameters associated with the metabolic syndrome are summarized in Table 2. The effect of the medium compared to the high fat and fructose allowance could not be measured for all parameters because either the sampling was associated with the killing of the animals and thus could only occur at the end of the experiment, or because for animal welfare reasons. During the "medium fat and fructose" period, blood could be taken only from a small number of randomly selected animals, the number of which was insufficient for a correct statistical evaluation. Thus, Figure 2 illustrates the time course of fecal and blood ethanol concentrations and associated selected parameters only for those rats, of which blood samples were available from both periods and for all parameters.

\section{Body Parameters}

While, as expected, the fat and fructose supply had a highly significant dose-dependent effect on weight development, neither the least-squares weight means nor the arithmetic means of the weight measured at the end of the experiment were significantly different in the W. confusa and control group (Table 2A).

\section{Liver-Associated Parameters}

The (relative) liver weight, determined as hepatic index $(\mathrm{HI} \%)$, was increased by $9.5 \%(\mathrm{p}=0.036)$ in the $W$. confusa group compared to the control group (Table 2B).

The serum levels of AST (GOT) and ALT (GPT) did not differ between the $W$. confusa and the control group, and were not (significantly) affected by the fat and fructose supply (Table 2B). 
A

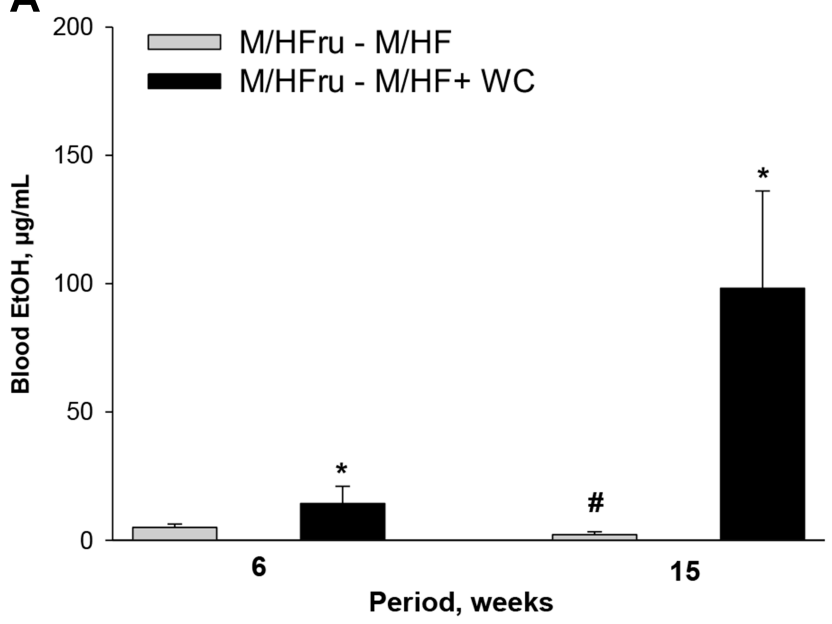

C

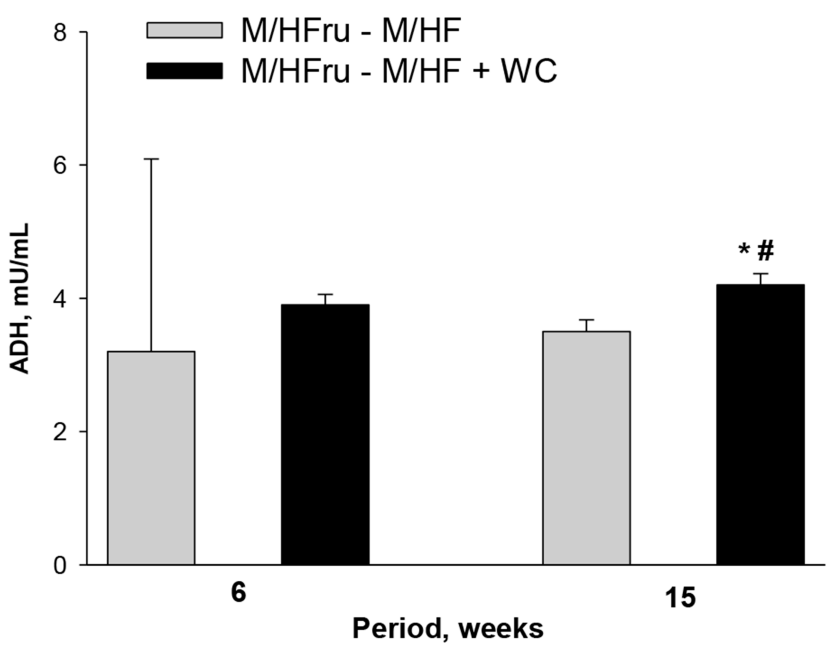

B

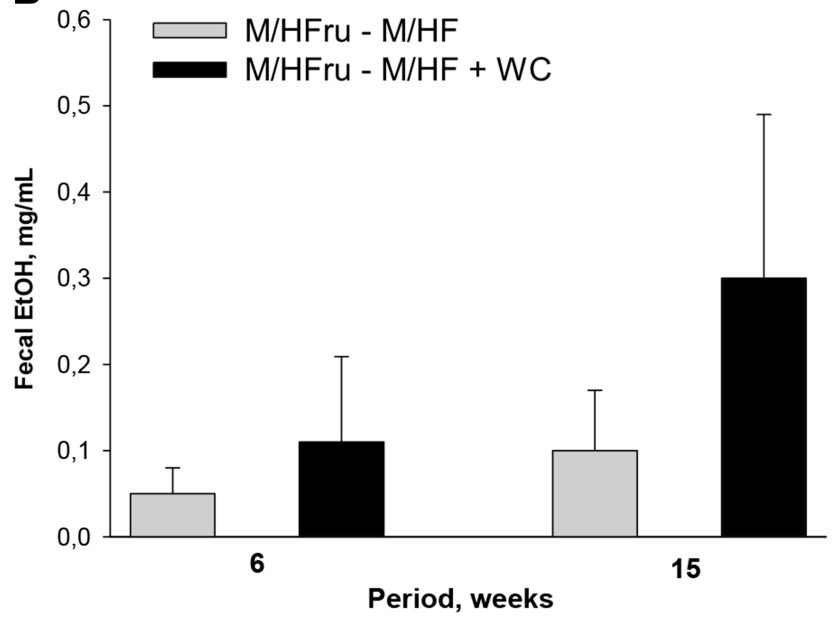

D

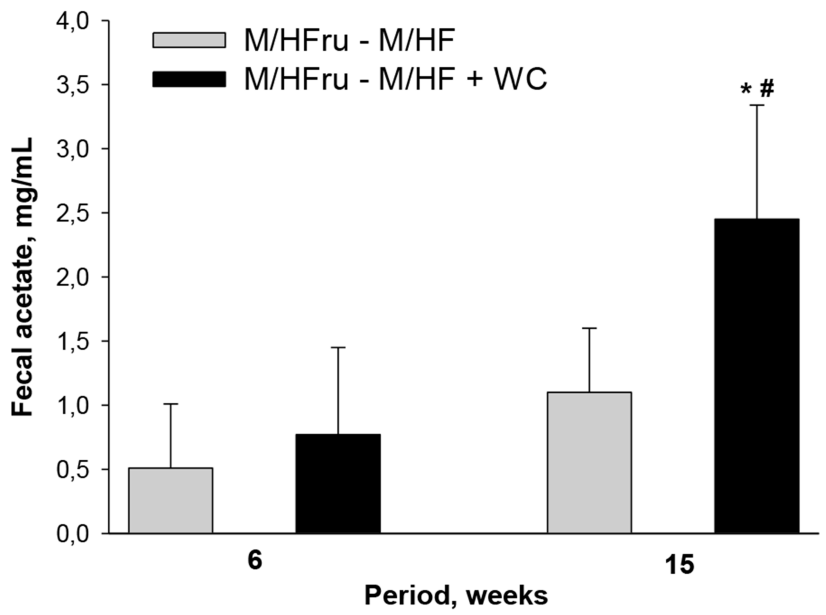

Figure 2 Effect of WC on ethanol concentrations in blood (A) and feces (B), on alcohol dehydrogenase activity (C) and on fecal acetate (D) in Wistar rats after six weeks of feeding a medium fructose and medium fat diet (MFru-MF) and after further nine weeks on a high fructose-high fat diet (HFru-HF). In this evaluation, only the ten rats were included, of which blood samples were available from both period and for all parameters. The figure shows mean values and standard deviations. The statistical comparison of the control and Weissella group and of the MFru-MF and HFru-HF periods was carried out by paired t-tests, separately for each period, or the control and Weissella group at the different periods, respectively.

Notes: *Denotes statistically significant $(P<0.05)$ differences from the corresponding values in rats fed a diet without WC. ${ }^{\#}$ Denotes statistically significant $(P<0.05)$ differences from the corresponding values of the MFru-MF period.

Abbreviations: $\mathrm{ADH}$, alcohol dehydrogenase; EtOH, ethanol; M/HFru-M/HF, medium (week 6) or high (week I5) fructose and medium (week 6) or high (week I5) fat diet; MFru-MF, medium fructose and medium fat diet; WC, Weissella confusa.

\section{Serum Parameters}

While $W$. confusa had no effect on serum glucose levels, mean serum triglyceride and cholesterol concentrations in the $W$. confusa group were increased by $27 \%$ and $18 \%$, respectively, compared to the control group (Table 2C). While the effect of $W$. confusa on serum cholesterol was highly significant $(p=0.003)$, the effect on serum triglycerides was significant only when using a one-sided statistical test $(\mathrm{p}=0.049)$. The latter seems justified, because randomized controlled trials and epidemiological studies show that alcohol intake results in either increased or unaltered serum triglyceride levels in male subjects. . $^{12,39,52}$

While virtually no ethanol was detectable in the blood in the control group (mean bEtOH $=0.115 \mu \mathrm{g} / \mathrm{mL}$ ), the value was highly significantly increased in the $W$. confusa group $(\mathrm{p}=0.003)$ to $75.1 \mu \mathrm{g} / \mathrm{mL}$ (Table $2 \mathrm{C}$ ).

\section{Fecal Parameters}

Fecal concentrations (or activities) of EtOH, alcohol dehydrogenase $(\mathrm{ADH})$ and the short-chain fatty acids acetate, propionate and butyrate were highly significantly 
A

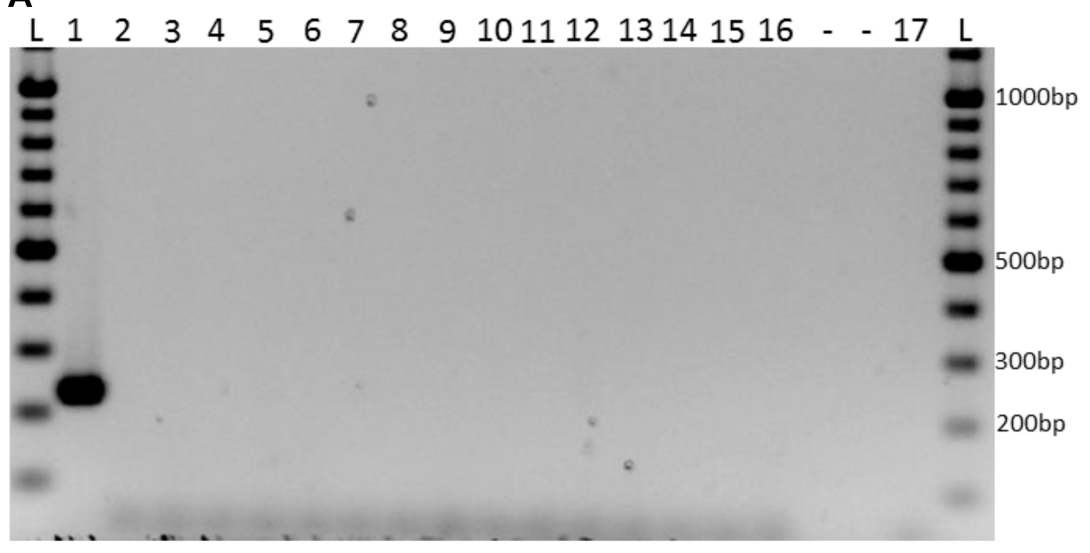

B

$\begin{array}{llllllllllllll}1 & 2 & 3 & 4 & 5 & 6 & 7 & 8 & 9 & 1011 & 121314151617181920 \quad \mathrm{~L}\end{array}$

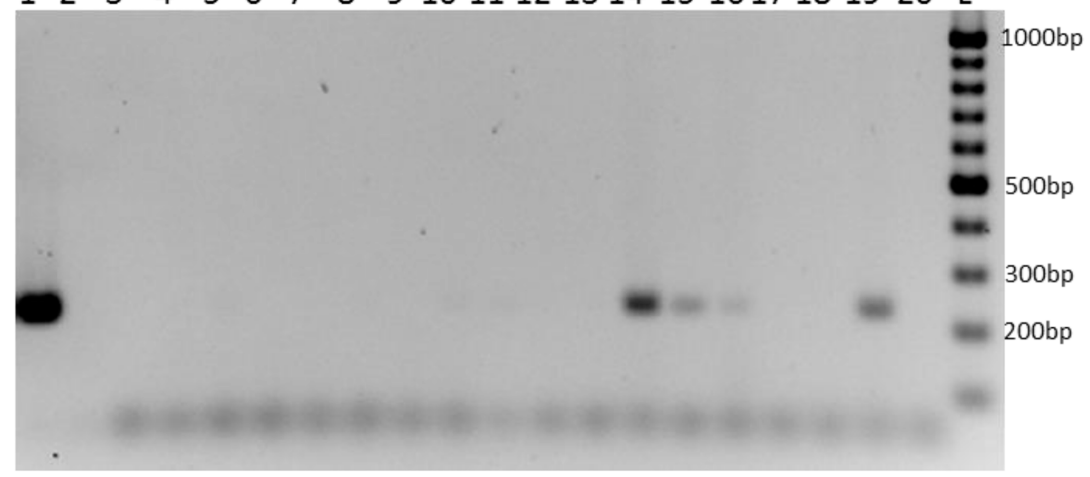

C

1223445667789101112131415161718 L -1920

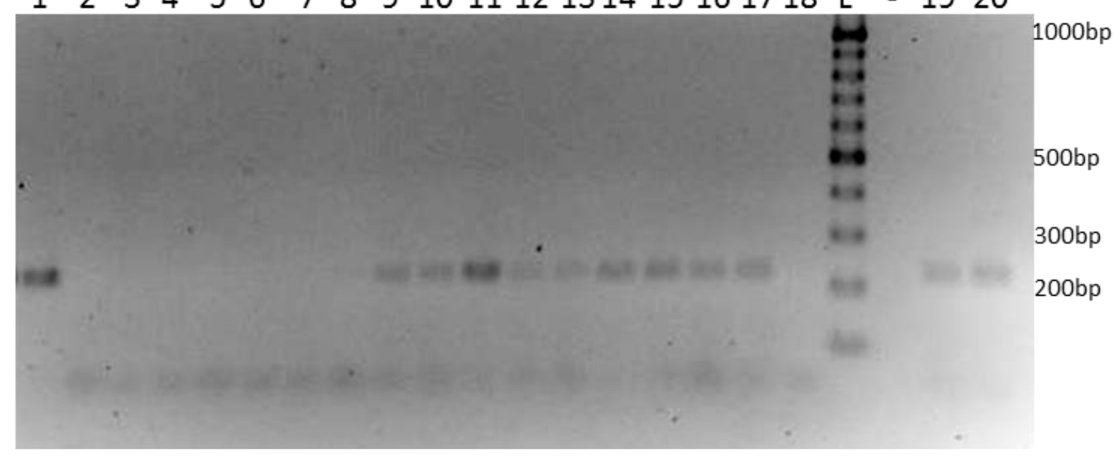

Figure 3 Amplification by PCR of Weissella confusae-specific lepA gene (225 bp) in male rats. (A) Rats were fed for 15 weeks ( 105 days) standard rat food (Ssniff NR*). Lane I: Positive control (2.3 ng WC genomic DNA), Lane 2-6: feces samples from rats at day 0, Lane 7-II: feces samples from rats at day 40, Lane 12-16: feces samples from rats at day 106, Lane 17: negative control, L: GeneRuler TM DNA Ladder Mix (Thermo Scientific, Fisher Scientific Co., Pittsburgh, USA). (B) Rats were fed for six weeks ( 42 days) the MFru-MF diet ( $28 \%$ fructose, $10 \%$ fat) and thereafter for nine weeks the HFru-HF diet (56\% fructose, $16 \%$ fat) according to a total intervention time of 15 weeks ( 105 days). Lane I: Positive control ( $2.3 \mathrm{ng}$ WC genomic DNA), Lane 2-7: feces samples were collected from rats at day 0, Lane 8-13: feces samples were collected from rats at the end of the MFru-MF diet feeding period (day 40), Lane I4-19: feces samples were collected from rats after the HFru-HF diet feeding period (day 106), Lane 20: negative control, L: GeneRulerTM DNA Ladder Mix (Thermo Scientific, Fisher Scientific Co., Pittsburgh, USA). (C) Rats were fed for six weeks ( 42 days) MFru-MF diet ( $28 \%$ fructose, $10 \%$ fat) mixed with WC. Thereafter, rats were accustomed to HFru-HF diet ( $56 \%$ fructose and $16 \%$ fat) mixed with WC for eight weeks according to a total intervention time of I5 weeks (I05 days). Lane I: Positive control (2.3 ng WC genomic DNA), Lane 2-7: feces samples were collected from rats at day 0, Lane 8-13: feces samples were collected from rats at the end of the MFru-MF diet feeding period (day 40), Lane 14-17 and lane 19-20: fecal samples were collected from rats after the HFru-HF diet feeding period (day 106), Lane 18: negative control, L: GeneRulerTM DNA Ladder Mix (ThermoScientific, Fisher Scientific Co., Pittsburgh, USA).

Note: *Product name (Ssniff NR) given by the provider (Ssniff, Soest, Germany).

Abbreviations: HFru-HF, high fructose-high fat; MFru-MF, medium fructose-medium fat; PCR, polymerase chain reaction; WC, Weissella confusa. 
increased in the $W$. confusa - compared to the control group (Table 2D). This increase was also associated with an increase in the fructose and fat content of the diet. The dose effect was significant.

In contrast, $W$. confusa had no significant effect on fecal lactate concentrations, and no significant dose effect of the fat plus fructose supplementation on this parameter was found (Table 2D).

\section{Detection of W. confusa in Rat Feces}

During intervention time (15 weeks) $W$. confusa was detected in the feces of all rats to whose diet $W$. confusa was added. Without such supplement, W. confusa was detected only in rats fed the HFru-HF diet, but not in rats fed standard rat food or the MFru-MF diet (Figure $3 \mathrm{~A}-\mathrm{C}$ and Table 2E).

\section{Discussion}

Intestinal microbiota has been shown to influence human health through stimulation of the immune system, improvement of digestion and absorption, biosynthesis of vitamins and lowering of gas distension. ${ }^{49,66}$ Gut microbiota and its metabolites have been described to have many effects on host metabolism leading to obesity and other traits of metabolic syndrome (MS). 5, 18,45,71 However, it is still a matter of discussion to which extent and by which mechanisms intestinal microbes may contribute.

Ethanol produced by the intestinal microbiota has been suggested to be one of the key factors in the development of obesity and other traits of the metabolic syndrome, such as NAFLD. ${ }^{6,21,64}$ Overgrowth of ethanol-producing bacteria in the intestine of obese patients induced the expression of ADH and cytochrome P450 2E1, which resulted in increased NADH levels as well as fatty acid synthesis leading to steatosis. ${ }^{6}$

High-fructose intake, as well as high-fat diet, is associated with overweight, insulin resistance, and other traits of the metabolic syndrome, incl. liver steatosis. ${ }^{26}$ Spruss and Bergheim ${ }^{59}$ and Lim et $\mathrm{al}^{46}$ suggested several mechanisms for the pathogenesis of NAFLD induced by highfructose intake based on increased translocation of bacterial endotoxins into the portal plasma resulting in activation of Kupffer cells, increased formation of proinflammatory TNF- $\alpha$, excess of mitochondrial acetylCoA being transformed into citrate, stimulation of de novo lipogenesis for fatty acids synthesis, and inhibition of hepatic lipid $\beta$-oxidation by excess of malonyl-CoA production.

Administration of high-fructose diet induced liver steatosis, NASH and inflammation in adipose tissue as well as markers of insulin resistance in rodents. ${ }^{13,42,59}$ Inflammation was suggested to be induced by disruption of gut barrier function and increased translocation of lipopolysaccharide (LPS). ${ }^{8,14,15}$ Disruption of gut barrier function and translocation of LPS is also induced in subjects with increased alcohol consumption ${ }^{7}$ and many other traits and mechanisms of the MSX are shared by high ethanol and by high-fructose consumption. Based on previous in vitro findings, we postulated that high-fructose diet may favor ethanol production by intestinal microbiota and hence explain common sequela: Indeed, ethanol was one of the important metabolites produced by intestinal heterofermentative lactobacilli, ie $W$. confusa from the fermentation of fructose in microbiological media. ${ }^{27}$ The inability to use fructose as electron acceptor (which would yield mannitol as one metabolite) was shown to be responsible for high ethanol amounts produced from the fermentation of fructose. Generally, heterofermentative lactobacilli like $W$. confusa need to keep the NADH/ NAD ratio balanced, when ATP is generated for energy production from fructose metabolism via xylulose-5-phosphate to lactate. This balance is attained by the recovery of NAD in the pathway from the common intermediate metabolite xylulose-5-phosphate via acetyl-phosphate to ethanol in $W$. confusa. While some heterofermentative lactobacilli, such as L. fermentum, are alternatively able to recover NAD by metabolizing fructose to mannitol and produce acetate instead of ethanol, $W$. confusa is not. ${ }^{3,27,74}$

Indeed, feeding of high fructose-high fat (HFr-HF) diet mixed with $W$. confusa resulted in significantly $(P<0.05)$ higher blood and fecal ethanol concentrations compared to control (Table 2C and D). The higher fecal ethanol was associated with higher fecal alcohol dehydrogenase (ADH) activity (Table 2D) indicating that at least part of $\mathrm{ADH}$ was of microbial origin. This finding is in line with results obtained by Tillonen et al (1999) ${ }^{62}$ who found that microbial counts of fecal slurries, levels of fecal ethanol and ADH activity were significantly decreased by the application of the antibiotic ciprofloxacin.

The finding of increased ethanol production on highfructose diet may explain how high-fructose intake is involved in the first step in the pathogenesis of NASH, namely disruption of gut barrier function. Ethanol can be metabolized to acetate and acetaldehyde. Acetaldehyde is 
suggested to lead to the formation of reactive oxygen species (ROS) resulting in increased intestinal permeability and inducing liver injury. ${ }^{25,48,50,60}$ Beside effects of acetaldehyde and ROS, further mechanisms of disruption of the intestinal barrier function by ethanol were suggested including an increase in inducible nitric oxide synthase (iNOS), activation of mast cells by acetaldehyde, alteration of microRNAs, and proliferation of Gram-negative bacteria and reduction of other bacterial species. ${ }^{20,31,54,60}$ Ethanol exposure was shown to diminish intestinal Akkermansia muciniphila abundance in both mice and humans and its abundance could be recovered in experimental ALD by oral supplementation. ${ }^{36}$ Dietary supplementation with A. muciniphila promoted intestinal barrier integrity and ameliorated experimental ALD. ${ }^{36}$ Akkermansia abundance is lower in overweight, hyperglycemia and hypercholesterolemia and associated with insulin sensitivity of the host. ${ }^{29,50,61}$ Akkermansia was shown to restore mucus thickness, which was reduced by dietary intervention in mice, to promote mucus production, ${ }^{29,38}$ and to improve gut barrier function and occludin expression by extracellular vesicles. ${ }^{17}$

The higher ethanol concentration in feces and peripheral blood found after $W$. confusa supplementation makes expect even higher blood levels in the portal vein draining the blood from the major parts of the large bowel directly to the liver. In contrast to absorption from ingested alcohol, ethanol from microbial metabolism in the large bowel is continuously produced until defecation. Thus, the peripheral blood levels are a result of a steady state between continuous intestinal ethanol production and degradation in the liver. Hence, the daily ethanol exposure and damage to the liver cannot be easily estimated by comparing peripheral ethanol levels induced by alcohol drinking with those found in this trial.

The higher blood levels of ethanol in the $W$. confusa group (Table $2 \mathrm{C}$ ) were associated with significantly ( $p<$ 0.003 ) higher levels of serum triglycerides and cholesterol (Table 2C) compared to control. This finding might suggest that ethanol can induce cholesterol and fatty acid synthesis because it acts as precursor of acetate after its oxidation. Acetate may be activated to acetyl-CoA which is used for cholesterol synthesis. Also, ethanol could activate the sterol regulatory element-binding protein (SREBP) which is responsible for fatty acids and cholesterol synthesis in hepatoma cell line. ${ }^{44,73}$ Generally, lipid homeostasis is altered by endogenous or chronic ethanol consumption in NAFLD or alcoholic fatty liver disease
(AFLD) leading to an increase in plasma triglycerides and fat content in triglyceride form in liver tissue. ${ }^{16,30,58}$

Hepatic index (HI)\% (ie the relative liver weight), which is a simple tool helping to identify NAFLD in humans ${ }^{43}$ and animal models, ${ }^{28,37}$ was significantly higher in the $W$. confusa - compared to the control group at the end of the intervention period (Table 2B), which might suggest that $W$. confusa induces liver steatosis.

Furthermore, ALT, which is regarded as biomarker for liver steatosis, ${ }^{50}$ was higher in the $W$. confusa - than in the control group $(p=0.001)$ at the end of the intervention period only after adjustment to the sampling time after 2 weeks (data not shown).

In contrast to $W$. confusa, mannitol positive heterofermentative lactobacilli like $L$. reuteri would - according to our suggestion - not result in the promotion of traits of the metabolic syndrome. Indeed, L. reuteri GMNL-263 could significantly reduce the hepatic index of rats fed highfructose diet. $^{41}$

Like lactose, fructose is not completely absorbed when ingested in excessive amounts. ${ }^{26}$ By that, it may enter lower parts of the intestine, where it can be fermented by microbiota via different pathways leading to the formation of different fermentation products, eg organic and shortchain fatty acids like lactate, succinate, acetate, propionate, butyrate, and ethanol and gases like hydrogen and carbon dioxide. ${ }^{11}$ Statistically significant increases in fecal concentrations of acetate, propionate and butyrate in the $W$. confusa group were observed, whereas the increase in concentrations of lactate was statistically not significant (Table 2D). The latter was to some extent unexpected, since lactate is a metabolite of the Embden-Meyerhof pathway produced by many intestinal bacteria fermenting fructose and also supposed to be produced in parallel with ethanol by $W$. confusa in our previous work. ${ }^{27}$ Lactate, however, may have been used as a substrate by colonic microbiota for producing different fecal SCFAs like acetate, propionate and butyrate. ${ }^{11,24,47,55}$

Concentrations of fecal acetate were significantly higher in the $W$. confusa group compared with the control (Table 2D). Higher levels of fecal acetate, a precursor for fatty acids and cholesterol, were associated with higher levels of plasma cholesterol and TG indicating increased activity of lipogenesis in the $W$. confusa group compared with the control. On the other hand, propionate, which was shown to inhibit cholesterol synthesis, ${ }^{68,69}$ was also significantly higher in the $W$. confusa group, in which the levels of cholesterol were higher. The overall increased 
lipogenesis may be explained by the relative proportion of acetate/propionate being about three-fold on a weight basis and even more so on a molar basis. Also, high concentrations of fecal SCFAs could increase peptide YY, which is responsible for decreasing the rate of intestinal transit, resulting in improved nutrient absorption. On the other hand, SCFAs were shown to increase levels of leptin which limits energy uptake. ${ }^{23,70}$ Recently, fecal SCFA was suggested to induce angiopoietin-like 4 (ANGPTL4) by activating peroxisome proliferator-activated receptor (PPAR) $\gamma$, resulting in reduced activity of the enzyme lipoprotein lipase (LPL), which catalyzes uptake of circulating lipids into tissues. ${ }^{5}$ Accordingly, ANGPTL4 overexpression raises circulating triglyceride levels and decreases fat storage in the tissues. ${ }^{1}$ Hence, by this mechanism, SCFA may have contributed to triglyceride elevation by $W$. confusa and counteracted against overweight and liver steatosis resulting in a complex interplay.

The fact that both the addition of $W$. confusa to rat food and a diet rich in fructose and fat resulted in the presence of $W$. confusa in the feces of the rats shows that, on the one hand, this species can survive gastrointestinal transit after oral administration, and, on the other hand, $W$. confusa in the feces of fat- and fructose-rich-fed rats can also originate from the autochthonous microbiota of the animals.

In combination with the results of previous investigations of our group, ${ }^{27}$ one may conclude that an increased abundance of $W$. confusa might promote NAFLD. Also, our results illustrate that under nutritional conditions like increased fructose and fat intake, heterofermentative lactobacilli negative for mannitol production might lead to an impairment of lipid metabolism and NAFLD. Assessment of markers, such as ethyl glucuronide and carbohydratedeficient transferrin, known to be associated with longterm alcohol abuse $\mathrm{e}^{40,53}$ and alcoholic fatty liver disease (AFLD) and histological investigation have to confirm whether this pathway links NAFLD and AFLD.

\section{Conclusion}

-Our findings suggest that heterofermentative lactic acid bacteria like $W$. confusa lacking the mannitol pathway and therefore being forced to metabolize fructose to ethanol indeed produce ethanol in amounts increasing fecal and peripheral blood ethanol concentrations in rats fed a highfructose, high-fat diet. This may explain why oral administration of $W$. confusa resulted in hyperlipidemia and may promote the development of NAFLD in this rat model. The production of ethanol from fructose by microorganisms like $W$. confusa also may explain why a high-fructose diet disrupts gut barrier function and enables LPS translocation. These results need to be confirmed in a better powered trial and to be substantiated by liver histology and by assessment of gut permeability, LPS levels, inflammatory markers and insulin resistance.

\section{Abbreviations}

$\mathrm{ADH}$, alcohol dehydrogenase; ALT (GOT), alanine transaminase; AST (GPT), aspartate aminotransferase; HF, high fat; HFru-HF, high fructose-high fat; HI\%, hepatic index; LSmeans, least-squares means; MFru-MF, medium fructosemedium fat; NAFLD, non-alcoholic fatty liver disease; NASH, non-alcoholic steatohepatitis; PCR, polymerase chain reaction; SCFA, short-chain fatty acid; TG, triglycerides.

\section{Acknowledgments}

This research was financially supported by DAAD-German Egyptian Research Long Term Scholarship program.

F. Repenning, M. Steinke, N. Sommer, H. Braun, S. HollerNiehus, V. Feiler, and J. Rohwer are gratefully acknowledged for their technical assistance. Northern Regional Research Laboratory (NRRL) is gratefully acknowledged for providing us with some intestinal lactic acid bacterial strains.

\section{Author Contributions}

All authors made substantial contributions to conception and design, acquisition of data, or analysis and interpretation of data; took part in drafting the article or revising it critically for important intellectual content; gave final approval of the version to be published; and agree to be accountable for all aspects of the work.

\section{Disclosure}

Dr Fouad M. F. Elshaghabee reports grants from MRI, during the conduct of the study. The authors report no other conflicts of interest in this work.

\section{References}

1. Alex S, Lange K, Amolo T, et al. Short-chain fatty acids stimulate angiopoietin-like 4 synthesis in human colon adenocarcinoma cells by activating peroxisome proliferator-activated receptor $\gamma$. Mol Cell Biol. 2013;33(7):1303-1316. doi:10.1128/MCB.00858-12

2. Amari M, Laguerre S, Vuillemin M, et al. Genome sequence of Weissella confusaLBAE C39-2, isolated from a wheat sourdough. $J$ Bacteriol. 2012;194(6):1608-1609. doi:10.1128/JB.06788-11

3. Axelsson L. Lactic acid bacteria: classification and physiology. In: Salminen S, von Wright A, Ouwehand A, editors. Lactic Acid Bacteria: Microbiological and Functional Aspects. 3rd rev. and exp ed. New York: Marcel Dekker, Inc.; 2004:1-66. 
4. Ayeni F, Adeniyi B, Ogunbanwo S, Samuel T, Nader-Macías M, Ruas-Madiedo P. Survival of Weissella confusa and Lactobacillus paracasei strains in fermented milks under cold storage and after freeze-drying. Milchwiss. 2011;66:61-64.

5. Bäckhed F, Ding H, Wang T, et al. The gut microbiota as an environmental factor that regulates fat storage. Proc Natl Acad Sci U S A. 2004;101(44):15718-15723. doi:10.1073/pnas.0407076101

6. Baker SS, Baker RD, Liu W, Nowak NJ, Zhu L. Role of alcohol metabolism in non-alcoholic steatohepatitis. PLoS One. 2010;5(3): e9570. doi:10.1371/journal.pone.0009570

7. Bala S, Marcos M, Gattu A, Catalano D, Szabo G. Acute binge drinking increases serum endotoxin and bacterial DNA levels in healthy individuals. PLoS One. 2014;9(5):e96864. doi:10.1371/journal.pone. 0096864

8. Bergheim I, Weber S, Vos M, et al. Antibiotics protect against fructose-induced hepatic lipid accumulation in mice: role of endotoxin. J Hepatol. 2008;48(6):983-992. doi:10.1016/j.jhep.2008. 01.035

9. Björkroth KJ, Schillinger U, Geisen R, et al. Taxonomic study of Weissella confusa and description of Weissella cibaria sp. nov., detected in food and clinical samples. Int J Syst Evol Microbiol. 2002;52(Pt 1):141-148. doi:10.1099/00207713-52-1-141

10. Björkroth JA, Dicks MTD, Endo A. The genus Weissella in lactic acid bacteria, biodiversity and taxonomy. In: Lactic acid bacteria: biodiversity and taxonomy. Holzapfel WH, Wood BJB, editors. Chichester: Wiley Blackwell; 2014:418-428.

11. Bourriaud C, Akoka S, Goupry S, Robins R, Cherbut C, Michel C. Butyrate production from lactate by human colonic microflora. Reprod Nutr Dev. 2002;42(Suppl. 1):S55-S63.

12. Brien SE, Ronksley PE, Turner BJ. Effect of alcohol consumption on biological markers associated with risk of coronary heart disease: systematic review and meta-analysis of interventional studies. $B M J$. 2011;342:636-645. doi:10.1136/bmj.d636

13. Bursać BN, Vasiljević AD, Nestorović NM, et al. High-fructose diet leads to visceral adiposity and hypothalamic leptin resistance in male rats - do glucocorticoids play a role? J Nutr Biochem. 2014;25 (4):446-455. doi:10.1016/j.jnutbio.2013.12.005

14. Cani PD, Amar J, Iglesias MA, et al. Metabolic endotoxemia initiates obesity and insulin resistance. Diabetes. 2007;56(7):1761-1772. doi: $10.2337 / \mathrm{db} 06-1491$

15. Cani PD, Amar J, Iglesias MA, et al. Selective increases of bifidobacteria in gut microflora improve high-fat-diet-induced diabetes in mice through a mechanism associated with endotoxaemia. Diabetologia. 2007;50(11):2374-2383. doi:10.1007/s00125-0070791-0

16. Carrasco MP, Jiménez-López JM, Segovia JL, Marco C. Comparative study of the effects of short- and long-term ethanol treatment and alcohol withdrawal on phospholipid biosynthesis in rat hepatocytes. Comp Biochem Physiol B Biochem Mol Biol. 2002;131(3):491-497. doi:10.1016/S1096-4959(02)00006-4

17. Chelakkot C, Choi Y, Kim DK, et al. Akkermansia muciniphila-derived extracellular vesicles influence gut permeability through the regulation of tight junctions. Exp Mol Med. 2018;50(2): e450. doi:10.1038/emm.2017.282

18. Clarke SF, Murphy EF, O'Sullivan O, et al. Targeting the microbiota to address diet-induced obesity: a time dependent challenge. PLoS One. 2013;8(6):e65790. doi:10.1371/journal.pone.0065790

19. Collins MD, Samelis J, Metaxopoulos J, Wallbanks S. Taxonomic studies on some Leuconostoc-like organisms from fermented sausages: description of a new genus Weissella for the Leuconostoc paramesenteroides group of species. J Appl Bacteriol. 1993;75: 595-603. doi:10.1111/j.1365-2672.1993.tb01600.x

20. Compare D, Coccoli P, Rocco A, et al. Gut-liver axis: the impact of gut microbiota on non-alcoholic fatty liver disease. Nutr Metab Cardiovasc Dis. 2012;22(6):471-476. doi:10.1016/j.numecd.2012. 02.007
21. Cope K, Risby T, Diehl AM. Increased gastrointestinal ethanol production in obese mice: implications for fatty liver disease pathogenesis. Gastroenterology. 2000;119(5):1340-1347. doi:10.105 3/gast.2000.19267

22. Daniel H, Gholami AM, Berry D, et al. High-fat diet alters gut microbiota physiology in mice. ISME J. 2014;8(2):295-308. doi:10.1038/ismej.2013.155

23. Delzenne NM, Cani PD. Interaction between obesity and the gut microbiota: relevance in nutrition. Annu Rev Nutr. 2011;31:15-31. doi:10.1146/annurev-nutr-072610-145146

24. Duncan SH, Louis P, Flint HJ. Lactate-utilizing bacteria, isolated from human feces, that produce butyrate as a major fermentation product. Appl Environ Microbiol. 2004;70:5810-5817. doi:10.1128/ AEM.70.10.5810-5817.2004

25. Elamin E, Masclee A, Troost F, Dekker J, Jonkers D. Activation of the epithelial-to-mesenchymal transition factor snail mediates acetaldehyde-induced intestinal epithelial barrier disruption. Alcohol Clin Exp Res. 2014;38(2):344-353. doi:10.1111/acer.12234

26. Elliott SS, Keim NL, Stern JS, Teff K, Havel PJ. Fructose, weight gain, and the insulin resistance syndrome. Am J Clin Nutr. 2002;76:911-922. doi:10.1093/ajcn/76.5.911

27. Elshaghabee FM, Bockelmann W, Meske D, et al. Ethanol production by selected intestinal microorganisms and lactic acid bacteria growing under different nutritional conditions. Front Microbiol. 2016;7:47-60. doi:10.3389/fmicb.2016.00047

28. Endo H, Niioka M, Kobayashi N, Tanaka M, Watanabem T. Butyrateproducing probiotics reduce nonalcoholic fatty liver disease progression in rats: new insight into the probiotics for the gut-liver axis. PLoS One. 2013;8(5):e63388. doi:10.1371/journal.pone.0063388

29. Everard A, Belzer C, Geurts L, et al. Cross-talk between Akkermansia muciniphila and intestinal epithelium controls diet-induced obesity. Proc Natl Acad Sci U S A. 2013;110 (22):9066-9071. doi:10.1073/pnas.1219451110

30. Feinman L, Lieber CS. Ethanol and lipid metabolism. Am J Clin Nutr. 1999;70(5):791-792. doi:10.1093/ajen/70.5.791

31. Ferrier L, Bérard F, Debrauwer L, et al. Impairment of the intestinal barrier by ethanol involves enteric microflora and mast cell activation in rodents. Am J Pathol. 2006;168:1148-1154. doi:10.2353/ajpath.2006.050617

32. Fukushima M, Nakano M. Effects of a mixture of organisms, Lactobacillus acidophilus or Streptococcus faecalis on cholesterol metabolism in rats fed on a fat- and cholesterol-enriched diet. $\mathrm{Br}$ J Nutr. 1996;76:857-867. doi:10.1079/BJN19960092

33. Fusco V, Quero GM, Stea G, Morea M, Visconti A. Novel PCR-based identification of Weissella confusa using AFLP-derived marker. Int J Food Microbiol. 2011;145:437-443. doi:10.1016/j. ijfoodmicro.2011.01.015

34. Fusco V, Quero GM, Cho G-S, et al. The genus Weissella: taxonomy, ecology and biotechnological potential. Front Microbiol. 2015;6:155. doi:10.3389/fmicb.2015.00155

35. Galle S, Schwab C, Arendt E, Gänzle M. ExopolysaccharideForming Weissella Strains as Starter Cultures for Sorghum and Wheat Sourdoughs. J Agric Food Chem. 2010;58:5834-5841. doi:10.1021/jf1002683

36. Grander C, Adolph TE, Wieser V, et al. Recovery of ethanol-induced Akkermansia muciniphila depletion ameliorates alcoholic liver disease. Gut. 2018;67(5):891-901. doi:10.1136/gutjnl-2016-313432

37. Hamad EM, Taha SH, Abou Dawood AG, et al. Protective effect of whey proteins against nonalcoholic fatty liver in rats. Lipids Health Dis. 2011;57:1-7.

38. Hänninen A, Toivonen R, Pöysti S, et al. Akkermansia muciniphila induces gut microbiota remodelling and controls islet autoimmunity in NOD mice. Gut. 2018;67(8):1445-1453. doi:10.1136/gutjnl-2017314508

39. Hannuksela MI, Räme M, Nissinen AET, et al. Effects of ethanol on lipids and atherosclerosis. Pathophysiology. 2003;10:93-103. doi:10. 1016/j.pathophys.2003.10.009 
40. Helander A. Biological markers in alcoholism. In: Fleischhacker WW, Brooks DJ, editors. Addiction Mechanisms, Phenomenology and Treatment. Vienna: Springer; 2003:15-32.

41. Hsieh FC, Lee CL, Chai CY, et al. Oral administration of Lactobacillus reuteri GMNL-263 improves insulin resistance and ameliorates hepatic steatosis in high fructose-fed rats. Nutr Metabol. 2013;10:1-14. doi:10.1186/1743-7075-10-35

42. Kawasaki T, Igarashi K, Koeda T, et al. Rats fed fructose-enriched diets have characteristics of nonalcoholic hepatic steatosis. $J$ Nutr. 2009;139(11):2067-2071. doi:10.3945/jn.109.105858

43. Kiage-Mokua BN, de Vrese M, Kraus-Stojanowic M, et al. Hypertriglyceridemia and CVD risk factors' reduction using Lapacho tea. Int J Pharm Phytopharmacological Res. 2018;8:39-45.

44. Lefèvre AF, DeCarli LM, Lieber CS. Effect of ethanol on cholesterol and bile acid metabolism. J Lipid. 1972;13:48-55.

45. Ley RE, Turnbaugh PJ, Klein S, Gordon JI. Microbiol ecology: human gut microbes associated with obesity. Nature. 2006;444 (7122):1022-1023. doi:10.1038/4441022a

46. Lim JS, Mietus-Snyder M, Valente A, Schwarz JM, Lustig RH. The role of fructose in the pathogenesis of NAFLD and the metabolic syndrome. Nat Rev Gastroenterol Hepatol. 2010;7:251-264. doi:10.1038/ nrgastro.2010.41

47. Marquet P, Duncan SH, Chassard C, Bernalier-Donadille A, Flint HJ. Lactate has the potential to promote hydrogen sulphide formation in the human colon. FEMS Microbiol Lett. 2009;299:128. doi:10.1111/ j.1574-6968.2009.01750.x

48. Medina J, Fernández-Salazar LI, García-Buey L, Moreno-Otero R. Approach to the pathogenesis and treatment of nonalcoholic steatohepatitis. Diabetes Care. 2004;27(8):2057-2066. doi:10.2337/ diacare.27.8.2057

49. Mengheri E. Health, probiotics and inflammation. J Clin Gastroenterol. 2008;42:S177-S178. doi:10.1097/MCG.0b013e31817eedc4

50. Naito Y, Uchiyama K, Takagi T. A next-generation beneficial microbe: akkermansia muciniphila. J Clin Biochem Nutr. 2018;63 (1):33-35. doi:10.3164/jcbn.18-57

51. Neuschwander-Tetri BA, Caldwell SH. Nonalcoholic steatohepatitis summary of an AASLD Single Topic Conference. Hepatol. 2003;37:1202-1219. doi:10.1053/jhep.2003.50193

52. Onat A, Hergen G, Dursunoglu D, et al. Associations of alcohol consumption with blood pressure, lipoproteins, and subclinical inflammation among Turks. Alcohol. 2008;42:593-601. doi:10.10 16/j.alcohol.2008.06.007

53. Palmer RB. A review of the use of ethyl glucuronide as a marker for ethanol consumption in forensic and clinical medicine. Semin Diagn Pathol. 2009;26:18-27. doi:10.1053/j.semdp.2008.12.005

54. Rao R. Endotoxemia and gut barrier dysfunction in alcoholic liver disease. Hepatology. 2009;50(2):638-644. doi:10.1002/hep.23009

55. Reichart N, Duncan SH, Young P, et al. Phylogenetic distribution of three pathways for propionate production within the human gut microbiota. ISME J. 2014;8:1323-1335. doi:10.1038/ismej.2014.14

56. Shoelson SE, Herrero L, Naaz A. Obesity, inflammation and insulin resistance. Gastroenterol. 2007;132:2169-2180. doi:10.1053/j. gastro.2007.03.059

57. Solga SF, Diehl AM. Non-alcoholic fatty liver disease: lumen-liver interactions and possible role for probiotics. J Hepatol. 2003;38: 681-687. doi:10.1016/S0168-8278(03)00097-7
58. Spinucci G, Guidetti M, Lanzoni E, Pironi L. Endogenous ethanol production in a patient with chronic intestinal pseudo-obstruction and small intestinal bacterial overgrowth. Eur J Gastroenterol Hepatol. 2006;18(7):799-802. doi:10.1097/01.meg.0000223906.55245.61

59. Spruss A, Bergheim I. Dietary fructose and intestinal barrier: potential risk factor in the pathogenesis of nonalcoholic fatty liver disease. $J$ Nutr Biochem. 2009;20:657-662. doi:10.1016/j.jnutbio.2009.05.006

60. Szabo G, Bala S. Alcoholic liver disease and the gut-liver axis. World J Gastroenterol. 2010;16(11):1321-1329. doi:10.3748/wjg.v16.i11.1321

61. Tilg H, Moschen AR. Microbiota and diabetes: an evolving relationship. Gut. 2014;63(9):1513-1521. doi:10.1136/gutjnl-2014-306928

62. Tillonen J, Homann N, Rautio M, Jousimies-Somer H, Salaspuro M. Ciprofloxacin decreases the rate of ethanol elimination in humans. Gut. 1999;44:347-352. doi:10.1136/gut.44.3.347

63. Van der Meulen R, Adriany T, Verbrugghe K, De Vuyst L. In vitro kinetic analysis of oligofructose consumption by Bacteroides and Bifidobacterium spp. indicates different degradation mechanisms. Appl Environ Microbiol. 2006;72:1006-1012. doi:10.1128/AEM.72. 2.1006-1012.2006

64. Volynets V, Küper MA, Strahl S, et al. Nutrition, intestinal permeability, and blood ethanol levels are altered in patients with nonalcoholic fatty liver disease (NAFLD). Dig Dis Sci. 2012;57:1932-1941. doi:10.1007/s10620-012-2112-9

65. Waldram A, Holmes E, Wang Y, et al. Top-down systems biology modeling of host metabotype-microbiome associations in obese rodents. J Proteome Res. 2009;8:2361-2375. doi:10.1021/pr8009885

66. Wallace TC, Guarner F, Madsen K, et al. Human gut microbiota and its relationship to health and disease. Nutr Rev. 2011;69:392-403.

67. Walter J, Hertel C, Tannock GW, Lis CM, Munro K, Hammes WP. Detection of Lactobacillus, Pediococcus, Leuconostoc, and Weissella Species in human feces by using group-specific PCR primers and denaturing gradient gel electrophoresis. Appl Environ Microbiol. 2011;67:2578-2585. doi:10.1128/AEM.67.6.2578-2585.2001

68. Wong V, Tse CH, Lam TT, et al. Molecular characterization of fecal microbiota in patients with nonalcoholic steatohepatitis-a longitudinal. PLoS One. 2013;8:e62885. doi:10.1371/journal.pone.0062885

69. Wu GD, Chen J, Hoffmann C, et al. Linking long term dietary patterns with gut microbial enterotypes. Science. 2011;334:105-108. doi:10.1126/science. 1208344

70. Xiong Y, Miyamoto N, Shibata K, et al. Short chain fatty acids stimulate leptin production in adipocytes through the $\mathrm{G}$ protein coupled receptor GPR 41. Proc Natl Acad Sci U S A. 2004;101 (4):1045-1050. doi:10.1073/pnas.2637002100

71. Xu J, Mahowald MA, Ley RE, et al. Evolution of symbiotic bacteria in the distal human intestine. PLoS Biol. 2007;5(7):e156. doi:10.13 71/journal.pbio.0050156

72. Yang YY, Lin HC, Huang YT, et al. Adaptive vasodilatory response after octreotide treatment. Am J Physiol. 2001;281:G117-G123.

73. You M, Fischer M, Deeg MA, Crabb DW. Ethanol induces fatty acid synthesis pathways by activation of sterol regulatory element-binding protein (SREBP). J Biol Chem. 2002;277:29342-29347. doi:10.1074/ jbc.M202411200

74. Zaunmüller T, Eichert M, Richter H, Unden G. Variations in the energy metabolism of biotechnologically relevant heterofermentative lactic acid bacteria during growth on sugars and organic acids. Appl Microbiol Biotechnol. 2006;72:421-429. doi:10.1007/s00253-006-0514-3 


\section{Publish your work in this journal}

Hepatic Medicine: Evidence and Research is an international, peerreviewed, open access journal covering all aspects of adult and pediatric hepatology in the clinic and laboratory including the following topics: Pathology, pathophysiology of hepatic disease; Investigation and treatment of hepatic disease; Pharmacology of drugs used for the treatment of hepatic disease. Issues of patient safety and quality of care will also be considered. The manuscript management system is completely online and includes a very quick and fair peer-review system, which is all easy to use. Visit http://www.dovepress.com/ testimonials.php to read real quotes from published authors.

Submit your manuscript here: https://www.dovepress.com/hepatic-medicine-evidence-and-research-journal 